\title{
How can delirium best be prevented and managed in older patients in hospital?
}

\author{
Jayna M. Holroyd-Leduc MD, Farah Khandwala MSc, Kaycee M. Sink MD MAS
}

Previously published at www.cmaj.ca

\section{The case}

You are asked to conduct a preoperative assessment of an 86-year-old woman recently admitted to hospital with a fractured right hip. She reports having fallen while getting out of her bathtub but denies any prior history of falls. She has no other injuries. She lives alone at home and was functioning independently before admission. Her medical history includes osteoporosis, mild cognitive impairment and cataracts. She has no history of cardiac or respiratory disease and no known cardiac risk factors. Her medications on admission include calcium carbonate, vitamin $D$ and etidronate. She does not drink alcohol. Her physical examination is remarkable for visual impairment, a fractured right hip and a Mini-Mental Status Examination score of 23/30. Results of her preoperative tests, including measurement of electrolytes, blood glucose, creatinine and complete blood count as well as an electrocardiogram, are unremarkable. Given her age and her history of mild cognitive impairment and visual impairment, you are concerned that she is at increased risk of postoperative delirium. What is the best way to prevent delirium? How should you manage the patient if she does become delirious?

$\mathrm{D}$ elirium occurs in $25 \%-65 \%$ of patients admitted to hospital because of hip fracture..$^{1,2}$ The prevalence of delirium is as high as $74 \%$ among surgical patients ${ }^{3}$ and $11 \%-42 \%$ among general medical in-patients. ${ }^{4}$ Delirium is defined as an acute disturbance of consciousness accompanied by a change in cognition or by development of a perceptual disturbance. ${ }^{5}$ It develops over a short period (hours to days), and its course tends to fluctuate. Delirium may be caused by a general medical condition, substance intoxication, substance withdrawal or multiple causes. ${ }^{5}$ In this article, we systematically review the evidence regarding the prevention and management of delirium among older patients in hospital.

Patients in whom delirium develops while in hospital have significantly worse outcomes than those who do not become delirious. Developing delirium in hospital has been associated with an increased risk of death, longer hospital stays, an increased risk of hospital-acquired complications, persistent cognitive deficits and increased rates of discharge to longterm care facilities. ${ }^{6-9}$ Delirium is also predictive of poor postoperative recovery of functional status and mobility among patients with hip fracture. ${ }^{710}$ Because sicker patients are more

\section{Key points}

- Multicomponent interventions that combine a comprehensive assessment and strategies targeting risk factors for delirium appear to be effective in preventing delirium in older in-patients.

- There is insufficient evidence to support pharmacologic interventions for the prevention or management of delirium in older in-patients.

- Multicomponent interventions aimed at managing delirium have not been found to decrease mortality or length of hospital stay.

likely to become delirious than those with fewer comorbidities, delirium may not be the only factor contributing to these adverse outcomes.

\section{What are the risk factors for delirium?}

Based on evidence from a prospective cohort of over 1300 surgical patients and a subsequent systematic review, there are preoperative factors that are associated with an increased risk of delirium after noncardiac surgery ${ }^{11,12}$ (Box 1). Similar independent risk factors were found among 281 medical patients 70 years of age or older at the time of hospital admission; these included visual impairment (relative risk [RR] 3.5, 95\% confidence interval [CI] 1.2-10.7), severe illness (RR 3.5, 95\% CI 1.5-8.2), preexisting cognitive impairment (RR 2.8, 95\% CI 1.2-6.7) and dehydration (high urea-to-creatinine ratio; RR 2.0, 95\% CI 0.94.6). ${ }^{13}$ Prospective data from 508 medical patients 70 years of age or older identified several hospital-related risk factors; these included the use of physical restraints (RR 4.4, 95\% CI 2.5-7.9), malnutrition (RR 4.0, 95\% CI 2.2-7.4), the use of a bladder catheter (RR 2.4, 95\% CI 1.2-4.7), the addition of more than 3 new medications (RR 2.9, 95\% CI 1.6-5.4) and any iatrogenic event (RR 1.9, 95\% CI 1.1-3.2).14 The cause of delirium was rarely isolated to just 1 factor; instead multiple precipitating factors contributed to the condition. ${ }^{14}$

From the Divisions of Geriatrics and General Internal Medicine (HolroydLeduc) and the Knowledge Translation Program (Holroyd-Leduc, Khandwala), University of Calgary, Calgary, Alta.; and the Section on Gerontology and Geriatric Medicine (Sink), J. Paul Sticht Center on Aging, Wake Forest University Baptist Medical Center, Winston-Salem, USA

CMAJ 2009.DOI:10.1503/cmaj.080519 
Box 1: Risk factors for delirium after noncardiac surgery* 11,12

- Age $\geq 70$ years (OR 3.3, 95\% Cl 1.9-5.9)

- Existing cognitive impairment (OR 4.2, 95\% Cl 2.4-7.3)

- Functional impairment (OR 2.5, 95\% Cl 1.2-5.2)

- Alcohol abuse (OR 3.3, 95\% Cl 1.4-8.3)

- Abnormal preoperative level of sodium, potassium or glucose (OR 3.4, 95\% Cl 1.3-8.7)

- Preoperative psychotropic drug use (OR not available)

- Depression (OR not available)

- Increased comorbidity (OR not available)

- Living in a long-term care facility (OR not available)

- Visual or hearing impairment (OR not available)

*Odds ratios (ORs) and $95 \%$ confidence intervals ( $\mathrm{Cls}$ ) are provided where available.

\section{Literature review}

Given the prevalence of delirium and its association with adverse health outcomes, it is important to prevent delirium among older patients admitted to hospital and to initiate appropriate management strategies when it does occur. We searched MEDLINE (using Ovid) from 1950 to October 2007 and EMBASE from 1980 to October 2007 to identify relevant studies. The search strategy included the terms "delirium," "confusion," "aged 65 or older," "hospitalization" and "in-patient," using the Cochrane randomized controlled trial filter and a systematic review filter. We also searched the Cochrane Database of Systematic Reviews and reviewed the references included in relevant systematic reviews. We identified English-language articles that addressed the prevention or management of delirium among adults aged 65 years or older in hospital. We accepted the definition for delirium used in the studies as long as one was described. We identified additional studies by searching the bibliographies of retrieved articles. Articles in languages other than English were excluded for logistic reasons. Additional details on the search methods are available in Appendix 1 (available at www.cmaj.ca/cgi/content/full/cmaj .080519/DC1).

The search yielded 408 citations ( 92 from MEDLINE and 316 from EMBASE). We excluded 30 non-English studies (7\% of the citations). We retrieved the full-text articles of 56 citations that met the initial inclusion criteria. A further 14 articles were identified through review of references cited in retrieved articles and Cochrane systematic reviews (Appendix 2, available at www.cmaj.ca/cgi/content/full/cmaj.080519 /DC1). After assessing the 70 articles, we excluded 59 for the following reasons: the article was a review article or editorial (24 studies), the study was not a randomized controlled trial (19), there were no relevant outcome data (10), the article described the protocol only (1), the article was a practice guideline (1), or the study population did not consist primarily of in-patients aged 65 years or older (4). The remaining 11 articles, published between 1987 and 2007, were included in our systematic review (Table 1). ${ }^{15-25}$

\section{How can delirium be prevented?}

We identified 8 trials that studied methods to prevent delirium in older patients in hospital (Table 1). Three trials involved multicomponent interventions $(n=646)$ among hip fracture patients. Five trials involved pharmacological interventions.

\section{Multicomponent interventions}

All 3 trials of multicomponent interventions for the prevention of delirium involved specialists in geriatrics and multicomponent strategies to target risk factors for delirium (Table 2). Two of the 3 studies conducted intention-to-treat analyses. The outcome assessors in all 3 trials were blinded. Two trials used the Confusion Assessment Method ${ }^{26}$ to diagnosis delirium; the third trial used a modified version of the Organic Brain Syndrome Scale. ${ }^{27}$ The Confusion Assessment Method is a tool designed for diagnosing delirium. It can be completed in less than 5 minutes, includes 4 criteria (acute onset and fluctuating course, inattention, disorganized thinking, and altered level of consciousness), is very accurate (sensitivity $94 \%$, specificity $90 \%$ ) and has high interobserver reliability (kappa $>0.8){ }^{26}$ The Organic Brain Syndrome Scale was designed to detect and monitor confusion. It consists of 2 parts (disorientation subscale and confusion subscale) and has excellent agreement with the Confusion Assessment Method. ${ }^{27}$

The multicomponent interventions appeared to be effective in preventing delirium among patients admitted to hospital with hip fracture (summary RR $0.75,95 \%$ CI $0.64-0.88$; $p$ for heterogeneity $=0.58$ ). The number needed to treat to prevent 1 case of delirium was 7 (95\% CI 4-20). In one of the trials, there were nonsignificant $(p>0.1)$ differences in baseline characteristics, including prefracture dementia and impairment in activities of daily living. ${ }^{23}$ In multivariable analyses to adjust for these differences, they found similar effect sizes, but the results were no longer significant.

In only 1 of the 3 studies did the intervention have a significant effect on postoperative length of hospital stay (28 days in the intervention group v. 38 days in the control group; $p=$ $0.03){ }^{22}$ However, the intervention in this study also involved extensive staff education and teamwork around other issues such as the prevention of osteoporosis, rehabilitation goals and discharge planning. There was no difference in discharge location between the intervention and control groups in the 2 studies reporting this outcome. ${ }^{22,23}$

Two studies reported on mortality. ${ }^{22,25}$ Only one trial found a significant decrease in hospital mortality $(0.6 \%[1 / 155]$ in the intervention group v. $5.5 \%$ [9/164] in the control group; $p=0.03) .{ }^{25}$ The intervention in this trial primarily involved a geriatric team comprising a geriatrician, a rehabilitation specialist and a social worker that helped the usual-care group of surgeons and orthopedic nurses coordinate and provide care. However, the details around the care provided by this team were not well documented. As for other secondary outcomes, the multicomponent interventions appeared to reduce the incidence of medical complications, including pressure ulcers, ${ }^{22,25}$ urinary tract infections, ${ }^{22}$ sleeping problems, ${ }^{22}$ nutritional complications ${ }^{22}$ and falls. ${ }^{22}$ Despite the limitations of these trials, there appears to be a role for multicomponent interventions to 
prevent delirium. There is further evidence in support of this from an earlier study involving 852 medical in-patients (RR $0.66,95 \%$ CI 0.46-0.95). ${ }^{28}$ In this trial, the intervention was clearly outlined and involved many of the same preventive strategies used in the 3 trials we included in our review. Although it is one of the largest and best known trials of the prevention of delirium, we did not include it because patient assignment was done using prospective matching instead of randomization.

Recent practice guidelines developed by the British Geriatrics Society in conjunction with the Royal College of Physicians of London recommend that patients at high risk of delirium be identified at the time of hospital admission and that prevention strategies be incorporated into their care plan (grade A evidence).$^{29}$ Clinical practice guidelines for the management of delirium in older people in Australia also agree that only hospital-based multicomponent preventive strategies currently have good evidence to support their use..$^{30}$

\section{Pharmacologic interventions}

There were 5 trials of pharmacologic interventions, 4 of which enrolled fewer than 100 patients (Table 1). Since no 2 trials used the same drug, we were unable to combine data for analysis, and thus each trial is described individually.

In a trial involving 57 patients with hip fracture, rates of delirium were examined between patients who received epidural anesthesia with prilocaine and epinephrine with or without bupivacaine $(n=28)$ and patients who received general anesthesia with thiopental, succinylcholine, atropine and halothane $(n=29) .{ }^{17}$ Delirium was assessed using the Organic Brain Syndrome Scale. The method of randomization was not described. Outcome assessors were blinded and intention-to-

Table 1: Details of randomized controlled trials included in the systematic review of interventions for the prevention and management of delirium among older patients* in hospital

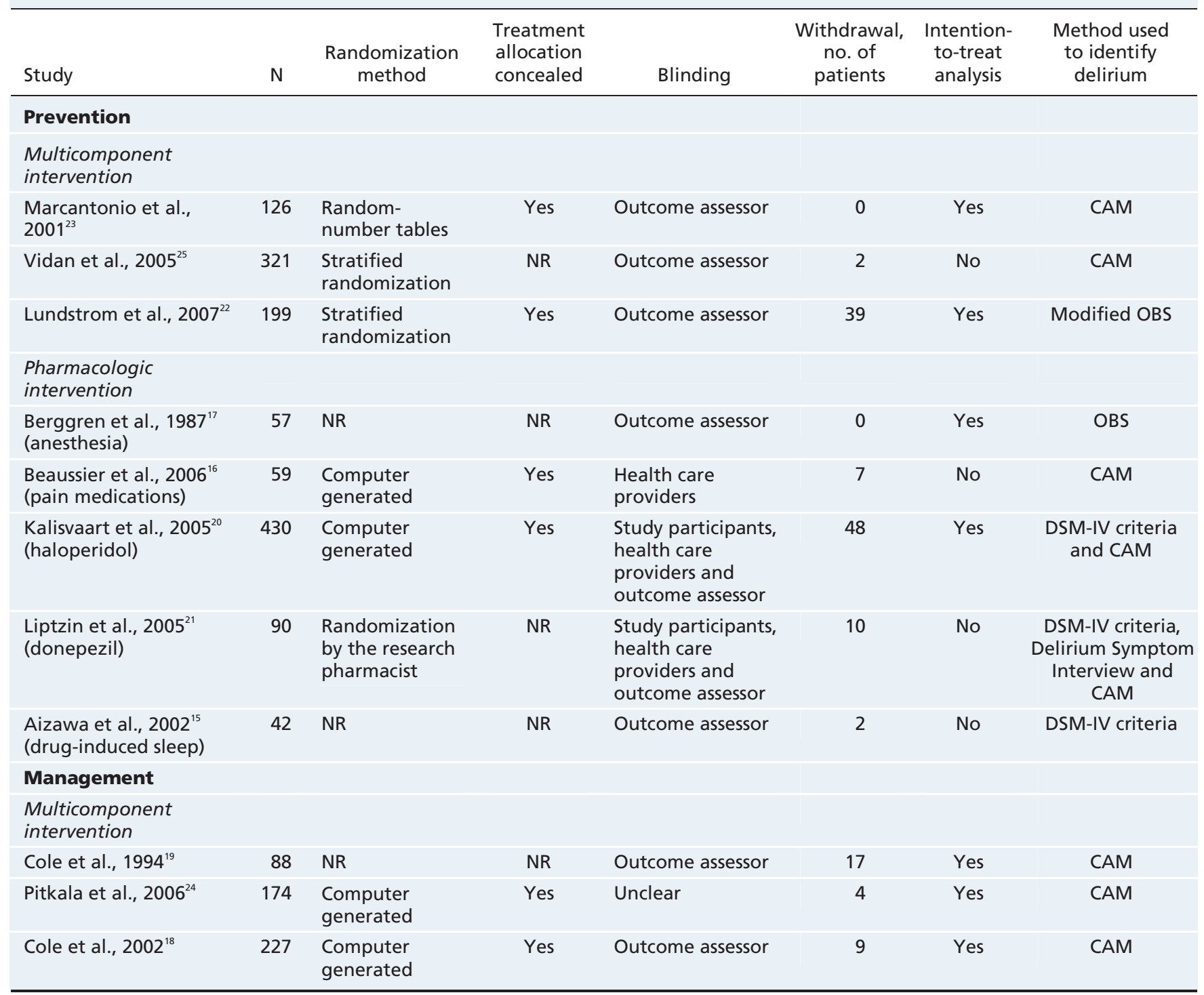

Note: $\mathrm{CAM}=$ Confusion Assessment Method, NR = not reported, OBS = Organic Brain Syndrome Scale.

*Age 65 years or more. 
treat analysis was completed. No significant difference in the rates of delirium were observed between the 2 study groups (50\% [14/28] in the epidural group v. 38\% [11/29] in the general anesthetic group).

One trial examined postoperative rates of delirium among 59 older patients undergoing surgical resection of colon or rectal cancers. ${ }^{16}$ The intervention group received preoperative intrathecal morphine at L4-L5 $(n=29)$; the control group received a preoperative subcutaneous injection of saline at L4-L5 $(n=30)$. Postoperatively both groups received propacetamol intravenously and patient-controlled morphine intravenously for breakthrough pain. Delirium was assessed using the Confusion Assessment Method. Randomization was computer generated; treatment allocation was concealed. The physicians in charge of the patients during the intraoperative

Table 2: Examples of strategies that targeted risk factors in multicomponent interventions for the prevention of delirium ${ }^{22,23,28,29}$

\begin{tabular}{|c|c|}
\hline Targeted risk factor & Strategy \\
\hline Cognitive impairment & $\begin{array}{l}\text { - Orientation protocols } \\
\text { - Provision of clocks and calendars }\end{array}$ \\
\hline Functional impairment & $\begin{array}{l}\text { - Early mobilization, including getting patient out of } \\
\text { bed regularly and as tolerated starting on } \\
\text { postoperative day } 1 \\
\text { - Daily physiotherapy with occupational therapy as } \\
\text { needed }\end{array}$ \\
\hline $\begin{array}{l}\text { Fluid and electrolyte } \\
\text { imbalances }\end{array}$ & $\begin{array}{l}\text { - Restoration of serum sodium, potassium and } \\
\text { glucose levels to normal limits } \\
\text { - Detection and treatment of dehydration or fluid } \\
\text { overload }\end{array}$ \\
\hline High-risk medications & $\begin{array}{l}\text { Discontinuation or minimization of use of } \\
\text { benzodiazepines, anticholinergics, antihistamines } \\
\text { and meperidine } \\
\text { - Modification of dosage or discontinuation of drugs } \\
\text { to minimize drug interactions and adverse effects }\end{array}$ \\
\hline Pain & $\begin{array}{l}\text { - Standing orders for acetaminophen use rather than } \\
\text { use as needed } \\
\text { - Treatment of breakthrough pain starting with low- } \\
\text { dose narcotics; avoidance of meperidine }\end{array}$ \\
\hline $\begin{array}{l}\text { Impaired vision and } \\
\text { hearing }\end{array}$ & $\begin{array}{l}\text { - Appropriate use of glasses, hearing aids and } \\
\text { adaptive equipment }\end{array}$ \\
\hline Malnutrition & $\begin{array}{l}\text { Ensurance of proper use of dentures, proper } \\
\text { positioning, assistance with eating if required and } \\
\text { use of supplements if required }\end{array}$ \\
\hline latrogenic complications & $\begin{array}{l}\text { - Removal of urinary catheter by postoperative } \\
\text { day } 2, \text { with screening for urinary retention and } \\
\text { incontinence } \\
\text { - Implementation of a skin-care program } \\
\text { - Bowel regimen to ensure bowel movements by } \\
\text { postoperative day } 2 \text { then every } 48 \text { hours } \\
\text { - Chest physiotherapy and supplemental oxygen if } \\
\text { indicated } \\
\text { - Appropriate anticoagulation therapy } \\
\text { - Screening and treatment of urinary tract infection }\end{array}$ \\
\hline Sleep deprivation & $\begin{array}{l}\text { - Unit-wide strategies to reduce noise } \\
\text { - Scheduling of medications and procedures to allow } \\
\text { for proper sleep } \\
\text { - Use of nonpharmacologic measures to promote } \\
\text { sleep }\end{array}$ \\
\hline
\end{tabular}

and postoperative periods were blinded to group assignment. Three patients were excluded in the intervention group: 1 each because of a major deviation in surgery, early postoperative abdominal sepsis, and aspiration pneumonia requiring intubation. Four patients were excluded in the control group: 2 because of a major deviation in surgery, 1 because of early postoperative abdominal sepsis and 1 because of death from pulmonary embolism. Of those remaining in the study, 9 patients in the intervention group and 10 in the control group had postoperative delirium.

The largest of the pharmacologic trials compared prophylactic haloperidol $(0.5 \mathrm{mg} 3$ times daily starting on admission and continued for 3 days postoperatively) and placebo in 430 patients with hip fracture at moderate or high risk of delirium..$^{20}$ Computer-generated randomization was conducted; treatment allocation was concealed. The research team and study patients were blinded to treatment allocation. Delirium was diagnosed on the basis of the DSMIV criteria and the Confusion Assessment Method. Once delirium was diagnosed, patients were given haloperidol or lorazepam, or both. Twenty of the 212 patients in the intervention group and 28 of the 218 in the control group dropped out. Among those who dropped out, data were missing for 35 patients (11 in the intervention group and 24 in the control group). The rates of delirium did not differ significantly between the 2 groups (15.1\% [32/212] in the haloperidol group v. $16.5 \%$ [36/218] in the placebo group; RR 0.91, 95\% CI 0.59-1.44). However, among the patients in whom delirium developed, those originally assigned to the haloperidol group had a shorter duration of delirium (5.4 days v. 11.8 days in the placebo group; $p<0.001)$ and a shorter hospital stay (17.1 days v. 22.6 days in the placebo group; $p<0.001$ ). There were no reports of drug-related extrapyramidal symptoms or sedation.

Donepezil $(5 \mathrm{mg} / \mathrm{d}$ for 14 days preoperatively and 14 days postoperatively), a cholinesterase inhibitor most commonly used in the treatment of dementia, was compared with placebo in a trial involving 90 patients undergoing elective total knee or hip replacement. ${ }^{21}$ Randomization was done by the research pharmacist. Study patients, health care providers and the outcome assessor were blinded to treatment allocation. Delirium was diagnosed on the basis of the DSM-IV criteria, the Delirium Symptom Interview $^{31}$ and the Confusion Assessment Method. The rate of delirium did not differ significantly between the study 
groups $(20.5 \%$ [8/39] in the donepezil group v. $17.1 \%$ [7/41] in the placebo group; $p=0.69$ ). The mean length of hospital stay did not differ either (4.4 days in the donepezil group v. 4.2 days in the placebo group; $p=0.09$ ).

A small trial involving 42 older patients undergoing surgical resection of gastric or colon cancer examined the use of intramuscular diazepam combined with a continuous intravenous infusion of flunitrazepam and pethidine administered from $8 \mathrm{pm}$ until $4 \mathrm{am}$ each night for 3 nights postoperatively. ${ }^{15}$ The investigators hypothesized that sleep disorders are a critical factor in the development of postoperative delirium and designed this protocol in an attempt to control disturbances in the sleep-wake cycle. The protocol was compared with usual care. The method of randomization was not documented. Delirium was diagnosed on the basis of the DSM-IV criteria by a psychiatrist who was blinded to group assignment. Two patients in the intervention group were excluded from analysis because of incomplete administration of the protocol. The rate of postoperative delirium was lower in the intervention group (5\% [1/20] v. $35 \%[7 / 20]$ in the control group; $p=0.02$ ). However, there was no statistically significant difference in length of hospital stay (25.6 days in the intervention group v. 29.9 days in the control group; $p=0.74$ ). The intervention led to morning lethargy in 8 patients.

Overall, there is currently insufficient evidence to support the use of any pharmacologic intervention for the prevention or management of delirium. However, further study into the role of antipsychotic agents in reducing the duration of delirium appears indicated.

\section{How should delirium be managed?}

We identified 3 trials (total 489 patients) that studied methods to manage delirium in medical in-patients (Table 1). All 3 trials involved a comprehensive geriatric assessment and multicomponent interventions targeted at precipitants of delirium. There were no pharmacologic trials identified.

The multicomponent interventions varied somewhat between studies but included strategies such as optimizing sensory input, orientation protocols, provision of familiar items and family presence, avoidance of restraints, encouragement of self-care, use of atypical antipsychotic agents for hyperactive and psychotic symptoms, use of nutritional supplements where indicated, screening for potentially treatable causes of cognitive impairment and comprehensive discharge planning. In 2 of the studies a geriatrician or geriatric psychiatrist assessed the patient; $;^{18,19}$ it is unclear who conducted the assessment in the third trial. ${ }^{24}$ All 3 trials conducted intentionto-treat analysis; the outcome assessors were blinded in 2 trials. In 2 trials, computer-generated randomization and concealed treatment allocation were used. The third trial did not describe the method of randomization. All 3 studies used the Confusion Assessment Method to diagnose delirium.

The multicomponent interventions for the management of delirium did not decrease mortality (summary RR 1.08, 95\% CI $0.81-1.44 ; p$ for heterogeneity $=0.77$ ). There was no effect on length of hospital stay (summary weighted mean difference 3.25 days, $95 \% \mathrm{CI}-2.85$ to 9.34 days; $p$ for hetero- geneity $=0.12){ }^{18,24}$ There was no impact on postdischarge dependency, ${ }^{18,19}$ function ${ }^{24}$ or the need for institutional care. ${ }^{24}$

\section{Gaps in knowledge}

Effectiveness studies are needed to determine whether multicomponent interventions for the prevention of delirium are feasible and cost-effective in everyday practice. There is insufficient evidence to support the use of any pharmacologic intervention for the prevention or management of delirium. Further study into the role of antipsychotic agents in reducing the duration of delirium would be useful.

\section{The case revisited}

After completing a comprehensive assessment, the physician elects to initiate a multicomponent preventive strategy that is targeted at the patient's risk factors for delirium, keeping in mind that not all the evidence around preventive strategies involved patients with hip fracture (Table 2). The physician chooses this option because the number needed to treat is only 7 , which indicates that this type of strategy has an excellent chance of reducing the risk of delirium in the patient.

High-risk medications such as benzodiazepines and anticholinergic agents are avoided. The patient receives a standing order for acetaminophen postoperatively, with low-dose narcotics (codeine 15-30 mg every 6 hours) as needed for breakthrough pain. A bowel regimen and appropriate anticoagulation are initiated following surgery. The patient's medication schedule is timed so as not to disturb her sleep through the night. The patient is oriented to place and time each morning. On postoperative day 1 , her urinary catheter is removed and she is helped out of bed. She receives daily physiotherapy starting 24 hours after surgery. Her oral intake is monitored, and her electrolytes and renal function are measured every 3 days. Appropriate strategies are used to compensate for her visual impairment, including ensuring that she wears her glasses. The patient is monitored closely for postoperative confusion using the Confusion Assessment Method on day 2 and 4. Delirium does not develop. After a short course of rehabilitation, she is discharged to her own home.

\section{Conclusion}

Although limited, the evidence currently available from randomized controlled trials of the prevention and management of delirium supports the implementation of multicomponent preventive strategies. In addition, the importance of consistently adhering to the various preventive strategies in these multicomponent interventions should be highlighted and considered when trying to implement these strategies into everyday clinical practice.

This article has been peer reviewed.

Competing interests: None declared.

Contributors: Jayna Holroyd-Leduc and Kaycee Sink were responsible for the conception and design of the study. Jayna Holroyd-Leduc, Kaycee Sink and Farah Khandwala were responsible for the acquisition, analysis and inter- 
pretation of the data. Jayna Holroyd-Leduc drafted the article. Kaycee Sink and Farah Khandwala critically revised the article. All of the authors approved the final version submitted for publication.

Funding: No external funding was received for this research. Kaycee Sink was supported by a Hartford Geriatrics Health Outcomes Award, funds from the Kulynych Center for Cognition Research, and funds from the WFU Claude D. Pepper Center (grant P30-AG21332).

\section{REFERENCES}

1. Inouye SK. Delirium after hip fracture: To be or not to be? J Am Geriatr Soc 2001;49:678-9.

2. Williams-Russo P, Urquhart BL, Sharrock NE, et al. Post-operative delirium: predictors and prognosis in elderly orthopedic patients. J Am Geriatr Soc 1992;40:759-67.

3. Dyer CB, Ashton CM, Teasdale TA. Postoperative delirium. A review of 80 primary data-collection studies. Arch Intern Med 1995;155:461-5.

4. Siddiqi N, House AO, Holmes JD. Occurrence and outcome of delirium in medica in-patients: a systematic literature review. Age Ageing 2006;35:350-64.

5. American Psychiatric Association. DSM-IV-TR diagnostic and statistical manual of mental disorders. 4th ed. Arlington (VA): The Association; 2000. p. 141-3.

6. Francis J, Kapoor WN. Prognosis after hospital discharge of older medical patients with delirium. J Am Geriatr Soc 1992;40:601-6.

7. Gustafson Y, Berggren D, Brannstrom B, et al. Acute confusional states in elderly patients treated for femoral neck fracture. J Am Geriatr Soc 1988;36:525-30.

8. Inouye SK, Rushing JT, Foreman MD, et al. Does delirium contribute to poor hospital outcomes? A three-site epidemiologic study. J Gen Intern Med 1998;13:234-42.

9. O'Keeffe S, Lavan J. The prognostic significance of delirium in older hospital patients. J Am Geriatr Soc 1997;45:174-8.

10. Marcantonio ER, Flacker JM, Michaels M, et al. Delirium is independently associated with poor functional recovery after hip fracture. J Am Geriatr Soc 2000;48:618-24.

11. Dasgupta M, Dumbrell AC. Preoperative risk assessment for delirium after noncardiac surgery: a systematic review. J Am Geriatr Soc 2006;54:1578-89.

12. Marcantonio ER, Goldman L, Mangione CM, et al. A clinical prediction rule for delirium after elective noncardiac surgery. JAMA 1994;271:134-9.

13. Inouye SK, Viscoli CM, Horwitz RI, et al. A predictive model for delirium in hospitalized elderly medical patients based on admission characteristics. Ann Intern Med 1993;119:474-81.

14. Inouye SK, Charpentier PA. Precipitating factors for delirium in hospitalized elderly persons. Predictive model and interrelationship with baseline vulnerability. JAMA 1996;275:852-7.

15. Aizawa K, Kanai T, Saikawa Y, et al. A novel approach to the prevention of postoperative delirium in the elderly after gastrointestinal surgery. Surg Today 2002;32:310-4

16. Beaussier M, Weickmans H, Parc Y, et al. Postoperative analgesia and recovery course after major colorectal surgery in elderly patients: a randomized comparison between intrathecal morphine and intravenous PCA morphine. Reg Anesth Pain Med 2006;31:531-8

17. Berggren D, Gustafson Y, Eriksson B, et al. Postoperative confusion after anesthesia in elderly patients with femoral neck fractures. Anesth Analg 1987:66:497-504.

18. Cole MG, McCusker J, Bellavance F, et al. Systematic detection and multidisciplinary care of delirium in older medical inpatients: a randomized trial. CMAJ 2002;167:753-9.

19. Cole MG, Primeau FJ, Bailey RF, et al. Systematic intervention for elderly inpatients with delirium: a randomized trial. CMAJ 1994;151:965-70.

20. Kalisvaart KJ, de Jonghe JF, Bogaards MJ, et al. Haloperidol prophylaxis for elderly hip-surgery patients at risk for delirium: a randomized placebo-controlled study. J Am Geriatr Soc 2005;53:1658-66.

21. Liptzin B, Laki A, Garb JL, et al. Donepezil in the prevention and treatment of post-surgical delirium. Am J Geriatr Psychiatry 2005;13:1100-6.

22. Lundstrom M, Olofsson B, Stenvall M, et al. Postoperative delirium in old patients with femoral neck fracture: a randomized intervention study. Aging Clin Exp Res 2007; 19:178-86.

23. Marcantonio ER, Flacker JM, Wright RJ, et al. Reducing delirium after hip fracture: a randomized trial. J Am Geriatr Soc 2001;49:516-22.

24. Pitkala KH, Laurila JV, Strandberg TE, et al. Multicomponent geriatric intervention for elderly inpatients with delirium: a randomized, controlled trial. J Gerontol A Biol Sci Med Sci 2006;61:176-81.
25. Vidan M, Serra JA, Moreno C, et al. Efficacy of a comprehensive geriatric intervention in older patients hospitalized for hip fracture: a randomized, controlled trial. J Am Geriatr Soc 2005;53:1476-82.

26. Inouye SK, van Dyck $\mathrm{CH}$, Alessi CA, et al. Clarifying confusion: the confusion assessment method. A new method for detection of delirium. Ann Intern Med 1990;113:941-8.

27. Jensen E, Dehlin O, Gustafson L. A comparison between three psychogeriatric rating scales. Int J Geriatr Psychiatry 1993;8:215-29.

28. Inouye SK, Bogardus ST Jr, Charpentier PA, et al. A multicomponent intervention to prevent delirium in hospitalized older patients. N Engl J Med 1999;340:669-76.

29. Potter J, George J. The prevention, diagnosis and management of delirium in older people: concise guidelines. Clin Med 2006;6:303-8.

30. Tropea J, Slee J, Brand CA. Clinical practice guidlines for the management of delirium in older people in Australia. Australas J Ageing 2008;27:150-6.

31. Albert MS, Levkoff SE, Reilly C, et al. The delirium symptom interview: an interview for the detection of delirium symptoms in hospitalized patients. $J$ Geriatr Psychiatry Neurol 1992;5:14-21.

Correspondence to: Dr. Jayna M. Holroyd-Leduc, Foothills Medical Centre, 1403-29th St. NW, Calgary AB T2N 2T9; jayna.holroyd-leduc@albertahealthservices.ca

\section{Send us your perspective}

\section{we like controversy.}

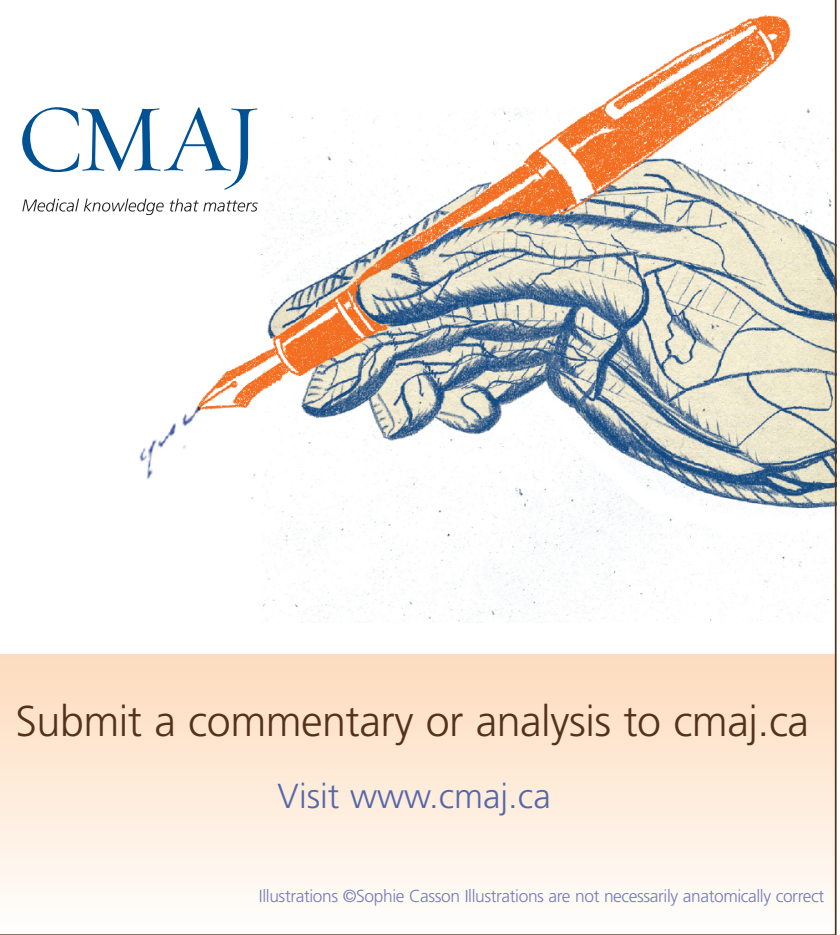

